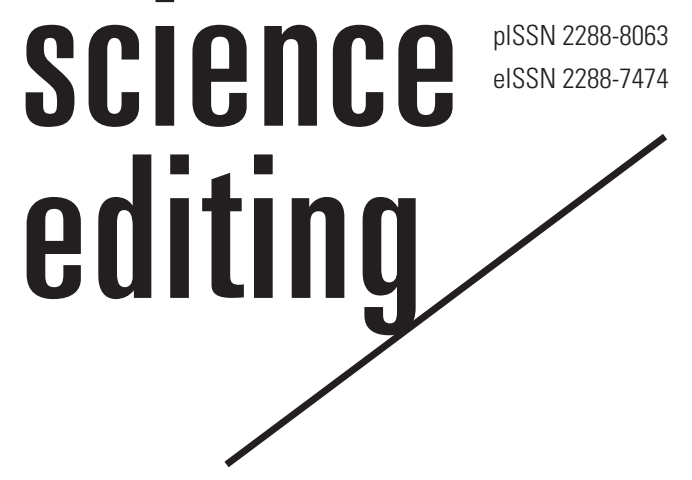

\title{
Issues advocated at 2019 annual meeting of the Council of Science Editors
}

\author{
Cheol-Heui Yun \\ Seoul, Korea \\ Date: May 4-9, 2019 \\ Venue: Hyatt Regency Columbus, Columbus, OH, USA \\ Organizer: Council of Science Editors
}

Department of Agricultural Biotechnology and Research Institute for Agriculture and Life Sciences, Seoul National University,

The 2019 annual meeting of the Council of Science Editors (CSE; Anna Jester, President) was held in Columbus, Ohio (May 4-9, 2019) with the theme and catch-phrase, 'the spirit of scientific publishing: inclusion, identity, technology \& beyond. Prior to the annual conference, there were one- and two-day short courses to provide editors and publication managers the opportunity to understand and collaborate on effective solutions for current and upcoming challenges. At the 2019 CSE annual meeting, the keynote address was given by Marjorie M. K. Hlava (President of Access Innovations, Inc.), and the plenary talk was given by Bernadette Melnyk (Vice President for Health Promotion, University Chief Wellness Officer, Dean of the College of Nursing, Ohio State University). Interesting sessions during the two-day meeting included General Data Protection Regulation (GDPR); Preprint submissions to journals: what's your policy; Manuscript Exchange Common Approach (MECA); and Creating and implementing a data policy among others.

Followings are the contents of the annual meeting in detail. The meeting was composed of short courses (May 4-5, 2019) and the CSE 2019 annual meeting (May 6-7, 2019). At the short courses just prior to the annual conference, there was a 1-day course for Advanced Publication Management. The publication management short course was a workshop for managing editors, production editors, and publication managers where they learned about up-to-date issues and had the opportunity to collaborate on effective solutions for current and upcoming challenges. The 2-day journal editors short course, designed for editors-in-chief and editorial members, was also offered. This course consisted of a comprehensive lecture and discussion of their roles and responsibilities (Fig. 1).

At the journal editors short course, David Crotty (Oxford University Press) suggested that while open access (OA) has advanced greatly and reached a new era of implementation, it is still in an evolutionary phase. He added that OA should indicate 'access and re-use' rather than 'open for the public.' He also compared and contrasted business models for publication including gold, green, and diamond/platinum OA. 

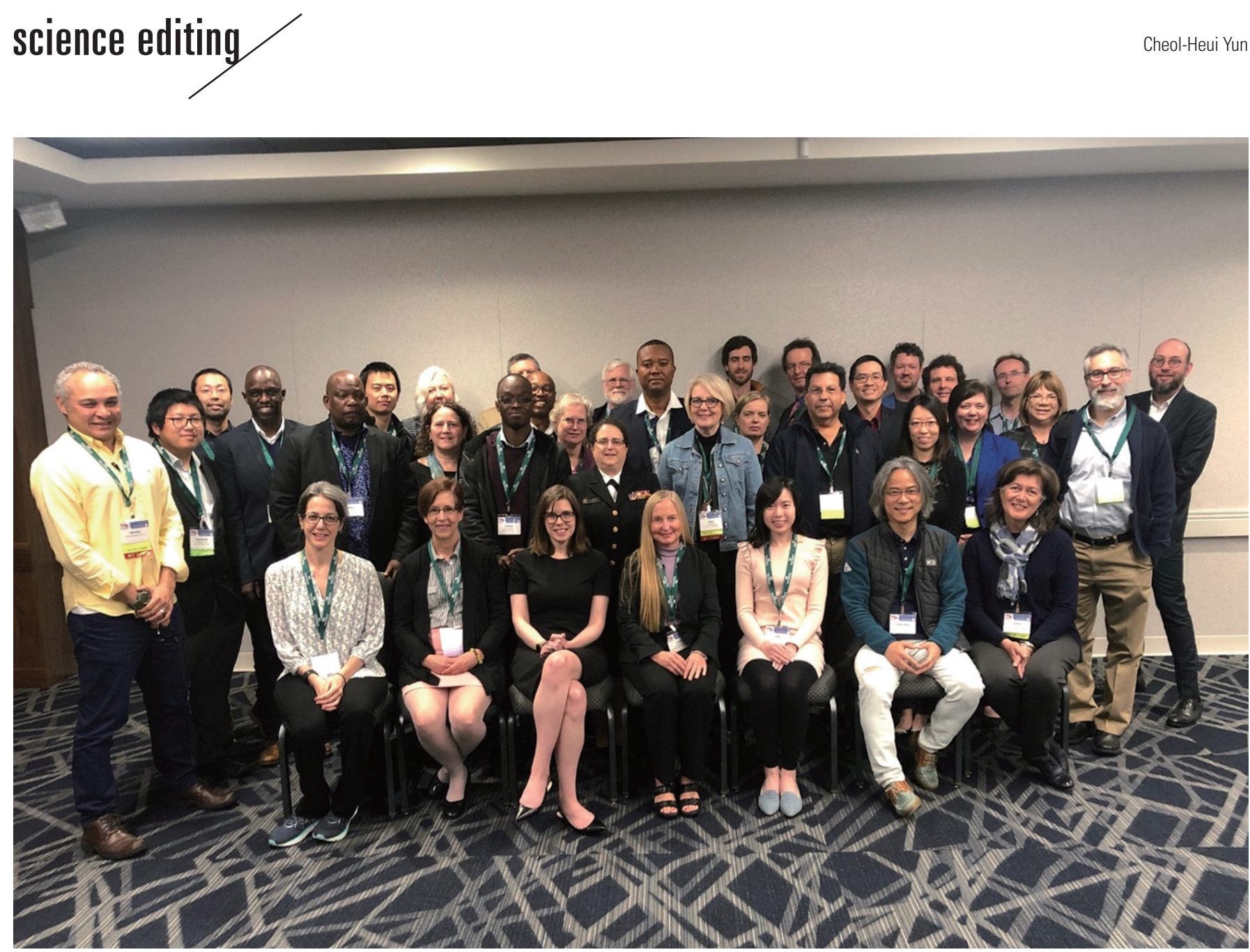

Fig. 1. Participants at journal editors short course, the 2019 annual meeting of the Council of Science Editors (Cheol-Heui Yun, the author, second from the right in the first row).

Briefly, problems and limitations with gold OA are as follows: author-centric rather than reader-centric, economic pressures to publish more rather than focusing on quality, cost often reduced by removing reader services, scale-dependent, artificially low article processing charge (i.e., average cost should not be assumed as the right price of all journals), productive institutes (e.g., some universities) that pay a lot more (e.g., the University of California spent about 35 million US dollars and would need about 75 million US dollars to move to gold $\mathrm{OA}$ ), shifts of the point of inequity from readers to the authors, phenomena that rich publishers get richer, possibility of disappearance of new journals and publishers in developing countries, and quality issues.

Problems and limitations with green OA are as follows: default embargo period of 12 months, wide variance of article half-life, different stakeholders with different needs, citation confusion due to versioning, and question of real OA.

Problems and limitations with diamond/platinum OA are as follows: unclearness on what to do when funds run out, easiness to make a new $\mathrm{OA}$, and hardness to maintain the publication.

After the session, I noticed that OA should not be confused with mega journal approaches and predatory journals. There are also new models of OA such as the consortia model, the subscribe to open model, and the bronze OA model.

One of the most popular sets of licenses developed by Creative Commons (CC), a non-profit organization, in conjunction with $\mathrm{OA}$, has focused on making creative works available for discovery and reuse. At the discussion, the reasons why CC BY-NC (Attribution-NonCommercial) and CC BY-ND (Attribution-NoDerivatives) have advantages over CC BY (Attribution) was discussed. Although it is an author's choice and preference of which type to use, CC BY-NC and CC BY$\mathrm{ND}$ are argued to be preferable because they protect author reputation and integrity of research, protect from unsavory commercial exploitation, protect intellectual property, provide licensing revenues, which reduce the financial burden, and provide a potential solution to the free-rider problem. It was 
noted that this issue might not be of high interest to authors as it would be a low priority for most of them.

According to Wikipedia, "Plan S is an initiative for openaccess science publishing that was launched by Science Europe on September 4, 2018. It is an initiative of 'cOAlition S', a consortium launched by major national research agencies and funders from twelve European countries. The plan requires scientists and researchers who benefit from state-funded research organizations and institutions to publish their work in open repositories or in journals that are available to all by 2020. The S stands for shock" [1]. Plan S in relation to OA was discussed based on various facts. National and international students tend to come to the famous lab in the area of interest where the principal investigator publishes in high impact factor journals. Plan S will not be supported by the US government. Chinese government welcomes Plan S, although there is no indication yet of its adoption since Chinese researchers like high impact factor journals regardless of the status of OA. Future directions for OA will include the following. The concept and mission statement of OA will be a valuable tool for societies. It must be carefully applied in a sustainable and responsible manner and there is still no perfect route. There are different approaches for different outcomes and no authors should be left behind.

I must say that OA is still just one of the models-not the answer or solution. Also, too much money is required regardless of the source for article processing charge. However, the direction suggested by OA is not incorrect, and yet the weight on how long it will take to gain a foothold will be different from country to country. It is seemingly true that we will get all this information not via libraries, with the result that there will be fewer libraries; otherwise, the role of the library will need to change.

Next, the peer review system was reviewed by Anne Coghill, a peer review analyst at the American Chemical Society (ACS). As we know, there is traditionally a single, double, and triple peer review system, and rather recently, the open peer review system has been introduced for its transparency. We also know that each model has merits and drawbacks. Ms. Coghill mentioned that ACS has not yet decided to transform from single peer review to other forms because of uncertainty. Specifically, the scientific community still seems conflicted. There is no guarantee of a better outcome. One major competitor (she did not mention the name) offers single and double options. Therefore, ACS decided to repeat the survey. Obviously, it is not an easy decision for any size journal or publisher to switch from one model to the other. Ms. Coghill introduced a very interesting feature, 'artificial intelligence in publishing and certain stage of peer review process' called 'review assistant'. She further described the artifi- cial intelligence projects at ACS for 'related content,' 'review recommendation,' and 'manuscript transfer'.

A few points about recruiting reviewers were described, specifically about being very careful when a person with a non-organization-based e-mail is contacted. Furthermore, when reviewers are recommended (by authors and colleagues), caution is needed. For instance, why the persons are recommended should be explained together with their expertise in the area. It is always a good idea to have a certain level of diversity in a reviewer pool, whether or not the manuscript that has been reviewed is accepted. It is also necessary to know what is different from the reviewers' decisions (and why), together with an editorial decision letter. It is probably the most important factor for journals and publishers to keep a good team of quality reviewers. Thus, certain type of awards for the reviewers should include publishing their names, invitation to the peer reviewer week, hosting a reception for reviewers at events, sending personalized cards or letters, providing certificates, and sending them calendars, luggage tags, and other types of swag.

Editors from the New England Journal of Medicine (NEJM) dissected the journal based on the data from 2017 and found that there have been constant changes (mobile, feedback, etc.) with their features and sharing capabilities (e.g., allowing each article to directly connect to Facebook, Twitter, LinkedIn, email, copy URL). Furthermore, they noticed that visits by device (e.g., smartphone) increased dramatically after changing the layout. The NEJM also provides a weekly eTOC (electronic table of contents). Although this is not a new feature at the NEJM, it is a good idea for publishers and journals that do not provide such an option for their readers. Of course, it is also important to ask about the authors' satisfaction. Some strategies the NEJM has recently tried out include visual abstract, "Instagram, Facebook, and Twitter" posting, quick references for clinicians, a social network service link in each article, and to make a well-fit presentation on each article, animation and video (one of the editors or editor-in-chief added a voice-over with caption).

The following information was provided by Christine Casey (Editor, MMWR Serials) after the roundtable discussion. Oxford University Press provides the content 'Measuring research: what everyone needs to know' by Cassidy R. Sugimoto and Vincent Larivière [2]. Embargo Watch provides various issues on 'keeping an eye on how scientific information embargoes affect new coverage' [3]. The strategies to recruit submissions were discussed and the Journal of Unmanned Vehicle Systems (contact Dominique Chabot) which was paired with a higher-impact journal for a 'joint virtual' issue of related fields was introduced [4].

The 2019 CSE annual meeting on May 6, 2019 started with 
a keynote address, entitled 'Project management, chickens, goats, and kids', by Marjorie M. K. Hlava (President of Access Innovations, Inc.). She discussed taxonomy and information systems and emphasized the importance of being a 'futurist.'

GDPR was introduced and discussed by Pamela Miller (special projects, NEJM), David Riley, and Heather Tierney, (Manager, Publication Ethics, ACS). GDPR is a regulation in the European Union (EU) that pertains to data protection and privacy for all individuals within the EU. The talk was interesting as it discussed (with an audience from elsewhere, not necessarily just from the USA and EU) the actual experience on the export of personal data outside the European Union. In particular, they provided actual cases and discussed the matters as follows. In case 1 on legal and ethical issues for retraction, what is the policy at the time of submission, including the GDPR agreement? Since everything is very international, it is very complicated, for example, to correspond with authors from other countries using data from many sources (or countries). In case 2, would the journal (editorial or editor-in-chief) allow a survey of corresponding authors (opt-in vs. opt-out, and no response)? Potential problems and limitations discussed included the fact that lawyers from different region seem to interpret the regulation differently. In case 3, medical records affiliated with EU patients get asked about liability and its proper use. There was an intense discussion about this issue on whether clients in EU would all be okay with this, and, if not, does the server location, the type of data collected, any other liability and legal obligations, and the time of the regulation (May 25, 2018) matter? The panel suggested the publishers and journals that have a lot of reviewer names on the list should opt-out, especially those who do not respond (whether or not their information is correct); specifically, personal information should be deleted immediately, including their e-mail, data, and document.

The session 'Preprint submissions to journals: what's your policy?' was led by Darla Henderson (Assistant Director, Open Access Programs, ACS Publications), Allison Leung (Senior Editor, Social Science Journals, Sage Publishing), and Laura Remis (Assistant Managing Editor, Science Advances, American Association for the Advancement of Science). The speakers focused on similar, but not the same, policies set by publishers and journals for preprint submission for publication, followed by changes and future prospects. Helen Atkins (Senior Manager, ACS), the session moderator, said that ACS has 36 different preprint policies and therefore there is no such thing as a 'gold standard, thus it is a case-by-case decision for journals and publishers. In fact, all preprint journals do have different policies. Ms. Atkins explained certain advantages of preprint journals, where some cases showed a lot more attention to preprint articles (e.g., ChemRxiv). She also appealed for the necessity of a direct journal transfer, although there are (potential) policy problems. Allison Leung explained that the SAGE preprint community is composed of approximately 1,000 journals and over 900 books. In addition, $83 \%$ of EU and US journals accepted preprints. She described not only submission guidelines and updated submission systems but also the need for continued education and refinement of policies.

At the end of the session, there was a discussion about the preprint system, where once an article is accepted in the preprint system (opt-in) but then none goes out system (opt-out). Ms. Remis made various points about the potential issues with preprints including scooped novel premature data, poor quality, embargo and press release conflict, and potential decline (while it remains in the preprint system). With this in mind, she mentioned that the AAAS (Science and its sister journals, Science Signaling, Science Translational Medicine, Science Advances, Science Immunology, and Science Robotics) allows preprints with its own policy. Indeed, one can find an article in the 'Policy Forum' of Science where editor-in-chief of Science introduced preprint [5]. At the end of the session, there was a Q\&A about the 'retraction policy' in preprint journals, as it does not seem to have any firm policy or regulation, but it does comply with the Committee on Publication Ethics (COPE). The other issue discussed was what level and how much verification of the manuscript should be done for simultaneous submission during the revision. The answer was that it has to be checked during every step of the processing.

One interesting session was the Update on the MECA Initiative by Joel Plotkin (CEO, eJournal Press), Eric Hall (Sr. Product Manager, HighWire Press), and Caroline Webber (business systems analyst, Aries Systems Corporation). The session description said that authors lose time and effort when their manuscript is rejected by a journal, and they have to repeat the submission process in subsequent journals. For reviewers, it is estimated that 15 million hours of researcher time is wasted each year repeating reviews. In addition, with the rise of preprint servers as an acceptable source of new submissions, there is a desire to have a seamless process to push papers from preprint servers to journals, including peer review information related to the preprint being transferred. All of these challenges could be addressed if journals, publishers, and preprint servers could transfer manuscripts between publications that use different submission-tracking systems. In 2017, a group of manuscript-management suppliers (Aries Systems, Clarivate, eJournal Press, and HighWire) took up this challenge and developed a common approach, including a set of guidelines and best practices that publishers, manuscript systems, and other players in the scholarly publishing ecosystem, such as preprint servers, authoring tools, and pro- 
duction services, can utilize so that communication between varied and diverse organizations can be more easily achieved.

In 2018, this initiative was accepted by the National Information Standards Organization (NISO), and a NISO working group was formed. This working group includes representatives from the original team of manuscript-management suppliers as well as representatives from the ACS, American Physical Society, Cold Spring Harbor Lab, eLife, IEEE, Green Fifteen, Jisc, Journal of Clinical Investigation, National Library of Medicine, Springer Nature, and Taylor \& Francis. The goal of the NISO working group is to make sure that the guidelines and recommended practices will be well thought out and universally accepted. The guidelines will continue to evolve to address new technologies and changes in data types and file formats that will likely emerge in the scholarly publishing infrastructure over the next few years. Tony Alves (Director of product management, Aries Systems Corporation) was the moderator of the session that touched on the historical background, current status, and upcoming issues. Panelists in this session saw the problems and opportunities on the issues with manuscripts going from journal to journal, and from preprint to journal. Caroline Webber described whatthey have done to set such activity and explained Megadata: JATS.xml; ID: Unique identifier; and TRANSMISSION: Secure FTP. Joel Plotkin showed some practical cases including EMBO, RUP (Rockefeller Univ Publ), CSHL, and Cellbiology network: JCB JCS MBoC for the transfer between sub-systems. Eric Hall explained the 'J2B or B2J (journal-2-bioRxiv bioRxiv-2-journal)' case where the first thing to do is to discuss and set what kind of information and data will be sent. There were questions from the audiences as follows. Q1. How much info could be sent? A1. Society may think, for example, the review and reviewer's name together with the review contents could make authors' lives easier. However, it could be a case-by-case decision. Q2. CC BY conditions its use (or differences). A2. It is only a coding matter at the end user (i.e., a personal matter). Besides, an author can use and modify xml and may not be the problem. Q3. CoI on information and data share. A3. It is possible.

On the second day of the CSE 2019 annual meeting (May 7, 2019), the plenary speaker, Bernadette Melnyk (Vice President for Health Promotion, University Chief Wellness Officer, Dean of the College of Nursing, Ohio State University) addressed the issue of work-life balance by sharing her personal difficulties and challenging life. She appealed strongly to the audience to be a positive person. She threw out a number of key questions and phrases to the audience to make the atmosphere vivid and make the audience feel involved. For instance, she asked: What would the last ten years of your life look like? Self-care is necessary in order to take care of others, and one should be strong enough to embrace this. Heart attack is the number one killer in the US (1 in 3), so the selfcare (one's behavior) is extremely important. Then, she asked everybody to stand up and follow her movements and emphasized the needs for 50 minutes of work combined with 10 minutes of non-sitting behavior. She also stressed that 20 minutes of activity improves brain circulation and functioning, stating that $80 \%$ of chronic diseases can be prevented by a healthy lifestyle. She proposed three keys for happiness: purpose, passion, and pride. She also asked everybody to stay aligned with their own dreams and passions.

The session entitled 'creating and implementing a data policy' might be important for publishers who want to have a data policy but have no roadmap to create one. This session was organized by inviting various stages of societies. Currently, publishers' signatories for a data policy number over 100 , including Science, PLoS, Elsevier, F1000, Nature, Wiley, and more. Although I was not able to follow the technical terms, there was an explanation about FAIR-Aligned [6] and Repository Finder developed by Datacite [7].

Michael Friedman explained 'data policy procedure' with conflicts or differences between FAIR and revised data policies based on software, model output, big dataset, and enforcement. Kerry Kroffe described the PLoS policy where authors must provide a data availability statement describing their compliance with PLoS policy; currently, about 115,000 papers are published in PLoS with data availability statement. Kerry Kroffe further emphasized the difficulties and expectation for 'data policy' by mentioning challenge created by broadening the scope, highly controversy at the time of inception, and considerable effort to implement. Alyson Weidmann (ACS) discussed 'issues of reproducibility in chemistry and biology' by first saying that a very high percentage of data are not reproducible (60 to $80 \%$ ). Thus, ACS set a task force for biological data guidelines for ACS (biological data guidelines policy) for enforcing proper statistics and explanation of methods. At the end of the session, there were questions as follows. Q1. Is there a data policy for medical science with clinical (patient) data? A1. Not yet, and one has to be very careful with the process. Q2. Is there a consistent structure, domain, and tagging cross other datasets? A2. Not really, if domain is different then, they may use a different repository.

The session entitled 'a picture's worth 1,000 words: disseminating research through graphical and visual abstracts' was also interesting and fresh. Graphical and visual abstracts have emerged as a powerful tool to disseminate and share research fast and accurately for busy scientists with the constant flow of new or advanced content. It is seemingly the best way to communicate complex research findings within a short time to reporters and to lay audiences in a more digestible format. 
Although one of the drawbacks is there is no repository (a database with hashtags has been discussed) for the graphical abstract yet.

Andrew Smith (production operations manager, Cell) presented a talk entitled 'graphical abstract: From the future to today, suggesting that this (i.e., graphical abstract) is created by an author, not peer-reviewed, for its first impression. He also introduced and explained, 'Cell press graphical abstract guidelines' in which the pdf version is available [8]. He added that a few benefits of this include that (1) ultimate sophistication becomes simple should be a huge benefit for author(s) and readers, (2) authors can use this in their presentation, and (3) on their SNS.

Mary Dott (MMWR) gave a talk on 'Using visual abstract at MMWR', introducing how the visual abstract and posters have evolved over time at MMWR. She mentioned that the changes during past years include competition with SNS in accordance with more people accessing information via digital devices than desktop computers. However, it was obvious that without some strategic planning, it would be near impossible to do without mentioning the cost. Ms. Dott talked about setting time line satisfaction, setting the target audience, the gradual impact (i.e., not an immediate effect after setting), consideration of visual abstracts in media, know-how (tips, tools, and tricks), engaging your team, embracing innovation, and endless review. She mentioned that the 'noun project' [9] would help.

A few poster presentations (there were seemingly not many poster presentations at this meeting) were displayed with the chance to meet with the authors. Rachel Winfield's poster, 'getting to know your authors: findings from the impact assessment and project appraisal's 2018 author survey, demonstrated that the composition and geography of editorial board members is varied yet concentrated on advanced economies, and that there is a positive relationship between the number of editors on an editorial board and the number of articles published by that journal. Furthermore, she showed that the larger an editorial board is, the more likely the board includes higher geographic diversity. Another poster, entitled 'factors on citation impact of publications in engineering, materials science, and medical journals (Hyunju Jang et al.), suggested that journal publishers and editors in Korea should attempt to attract more publications by foreign authors to increase their visibility and likelihood of being cited. Another poster, 'transpose landscape study on journal preprint and peer review policies (Jessica Polka et al.), highlighted the need for journals to clearly and explicitly state policies on their websites. While authors and reviewers may possess tacit knowledge about peer review or pre-printing practices within their fields, lack of explicit information may inhibit practices or expose re- searchers to risk.

Overall, the meeting was informative and well-organized, although I left with a feeling of fatigue from traveling a long distance. I also found it impossible to attend simultaneous and parallel sessions, and it was too dynamic to have time to chat with the speakers. However, needless to say, I was able to strengthen my grasp of new and old issues on scientific publishers and journals.

\section{Conflict of Interest}

Cheol-Heui Yun has served as an editorial board member of the Science Editing since 2014 but has no role in the decision to publish this article. Except for that, no potential conflict of interest relevant to this article was reported.

\section{Acknowledgments}

This work was supported partially by the Korean Council of Science Editors and by the National Research Foundation (NRF-2018R1A2B2006793), Republic of Korea.

\section{References}

1. Wikipedia. Plan S [Internet]. [place unknown]: Wikipedia [cited 2019 Jul 1]. Available from: https://en.wikipedia. org/wiki/Plan_S

2. Sugimoto CR, Lariviere V. Measuring research: what everyone needs to know. London: Oxford University Press; 2018.

3. Embargo Watch. Keeping an eye on how scientific information embargoes affect news coverage [Internet]. [place unknown]: Embargo Watch [cited 2019 Jul 1]. Available from: https://embargowatch.wordpress.com

4. Canadian Science Publishing. Journal of unmanned vehicle systems virtual issue [Internet]. Ottawa: Canadian Science Publishing [cited 2019 Jul 1]. Available from: https:// www.nrcresearchpress.com/journal/juvs-vi

5. Berg JM, Bhalla N, Bourne PE, et al. Preprints for the life sciences. Science 2016;352:899-901. https://doi.org/10.1126/ science.aaf9133

6. Coalition for Publishing Data in the Earth and Space Sciences (COPDESS). Enabling FAIR Data Project [Internet]. [place unknown]: Coalition for Publishing Data in the Earth and Space Sciences [cited 2019 Jul 1]. Available from: http://www.copdess.org/enabling-fair-data-project/

7. DataCite. Repository finder: a pilot project of the Enabling FAIR Data Project [Internet]. [place unknown]: DataCite [cited 2019 Jul 1]. Available from: https://repositoryfinder. datacite.org/ 
8. Cell Press. Cell Press graphical abstract guidelines [Internet]. Amsterdam: Elsevier [cited 2019 Jul 1]. Available from: https://www.cell.com/pb/assets/raw/shared/figureguidelines/GA_guide.pdf
9. Icons for everything [Internet]. [place unknown]: Noun Project [cited 2019 Jul 1]. Available from: https://thenounproject.com/ 